Andrews University

Digital Commons @ Andrews University

Honors Theses

Undergraduate Research

$4-30-2019$

\title{
A PFA Study: Exploring the Relationship of Anxiety and Self- Esteem with Expressive Writing
}

Abraham J. Gothong Walayat

Andrews University, abrahamw@andrews.edu

Follow this and additional works at: https://digitalcommons.andrews.edu/honors

Part of the Social and Behavioral Sciences Commons

\section{Recommended Citation}

Walayat, Abraham J. Gothong, "A PFA Study: Exploring the Relationship of Anxiety and Self-Esteem with Expressive Writing" (2019). Honors Theses. 212.

https://dx.doi.org/10.32597/honors/212

https://digitalcommons.andrews.edu/honors/212

This Honors Thesis is brought to you for free and open access by the Undergraduate Research at Digital Commons @ Andrews University. It has been accepted for inclusion in Honors Theses by an authorized administrator of Digital Commons @ Andrews University. For more information, please contact repository@andrews.edu. 
J. N. Andrews Honors Program

Andrews University

HONS 497

Honors Thesis

A PFA Study: Exploring the Relationship of Anxiety and Self-Esteem with Expressive Writing

Abraham J. Gothong Walayat

$4 / 30 / 2019$

Advisor: Dr. Harvey J. Burnett, Jr.

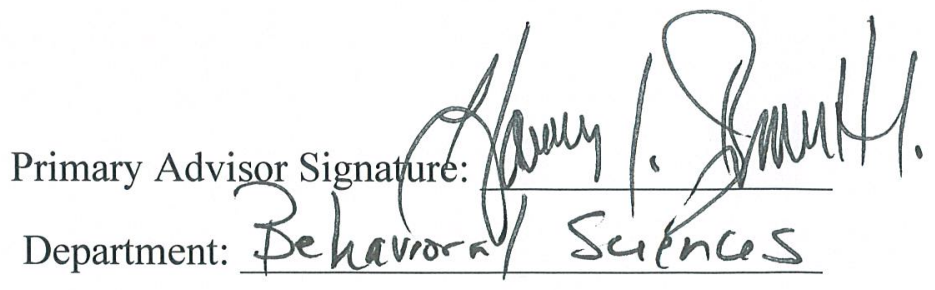




\begin{abstract}
Research has indicated that expressive writing is thought to be beneficial by decreasing inhibitions, but what was still unknown is how the use of self-esteem words could moderate the changes in anxiety levels. This study examined the efficacy of Expressive Writing on anxiety levels across time (baseline, immediately post treatment, 30 minutes post, and 15 days post) compared to a Superficial Writing control condition. This study also examined how the use of self-esteem words moderated anxiety levels. Subjects $(N=31)$ were drawn from the Andrews University Behavioral Science Research Subjects Pool and were randomly assigned to either an Expressive Writing experimental condition or a Superficial Writing control condition. Subjects also completed a demographic questionnaire and the State Trait Anxiety Inventory (State Anxiety scale). Self-esteem words were analyzed using the Linguistic Inquiry and Word Count (LIWC) program. Results from a repeated measures ANOVA revealed a significant change in anxiety levels across time for each condition, but did not indicate a significant difference between the two conditions. Results from an ANOVA also did reveal a significant interaction between anxiety levels and self-esteem, but the necessary level of self-esteem was different among subjects. The findings of this study would suggest that engaging in a writing activity is more helpful in decreasing anxiety levels over time, while the use of self-esteem words seem to have a small buffering effect.
\end{abstract}


A PFA Study: Exploring the Relationship of Anxiety and Self-Esteem with Expressive Writing

This study was based off of an on-going broader study examining the efficacy of Psychological First Aid (PFA) on anxiety, mood, and self-esteem compared to an expressive writing condition across time utilizing a treatment and control group. Research has indicated that expressive writing is thought to be beneficial by decreasing inhibitions, but what was still unknown was how the use of self-esteem words can moderate the changes in anxiety levels (Burling, et al, 1992; Pennebaker, 1990). The purpose of this study was to examine the differences in anxiety levels across time along with the number of self-esteem related words used through an expressive writing task, and how usage of self-esteem words moderated this effect.

It must be noted that this study was also collecting data for a larger parent study on PFA. As for the variables, expressive writing has been extensively studied by James W. Pennebaker. Expressive writing involves writing personally and emotionally without regards to form or grammar, and can benefit mental, physical, and psychological health (Pennebaker, 1990). To be effective, expressive writing has to be meaningful, impactful, and cathartic to the individual, so immediately after, expressive writing was correlated with slightly higher levels of anxiety directly after the activity, but was associated with long, strong decreases in anxiety over time. This study was largely based off of Pennebaker's work, but there is little research that has correlated the effects of expressive writing with the methods and program of Psychological First Aid. The hypothesis was largely built from Pennebaker's work, so it was believed that there would be a sharp increase in anxiety levels immediately after the expressive writing task, but those anxiety levels would eventually decrease, leading to long, strong decreases in anxiety levels overall (Pennebaker, 1990). In addition, it was likely that self-esteem might play a role in how the anxiety levels decrease, because individuals with self-esteem were correlated with 
having higher levels of resilience against negative events in life (De Jong, 2001). In this way, individuals with high levels of self-esteem should show even stronger, steeper decreases in anxiety due to their ability to "bounce back" from negative events more easily (De Jong, 2001; Pennebaker, 1990). A graph of the hypothesis suggests curvilinear changes in anxiety across time (see Appendix A).

Self-esteem is one's emotional self-worth, and high levels of self-esteem are often correlated with higher resilience and stronger feelings of control in one's life (De Jong, 2001). Therefore, because individuals who have higher self-esteem show higher levels of resilience, it was possible to infer that their anxiety levels should be additionally protected from the increasing anxiety. As such, there should be a lower spike during the post-test anxiety increase (Pennebaker, 1990). If the results are statistically significant, then a moderation analysis must be run on the self-esteem levels across participants to see if higher self-esteem levels are truly correlated with stronger decreases in anxiety levels.

Lastly, anxiety can be seen as the body's stress response, typically in response to issues that the individual does not have the power to act on, or simply feels that he or she do not want to think about (Byrne, 2000). Anxiety, in addition, can be considered from an abnormal psychology point of view. What this entails is that individuals who go through excruciatingly traumatic experiences have self-reported feeling "beside themselves" or that they are watching what is happening to themselves, like a play. This, it was hypothesized, is to decrease the amount of psychological damage and stress that is caused by trauma. Using this theory, it is possible that anxiety follows the same methods, just on a much smaller scale. That is, individuals who are anxious might disembody themselves from the events that are causing stress. While on a lesser scale than trauma, it could likely lead to feelings of uneasiness or forced repression of those 
events in an attempt to keep mood elevated. However, this "bottling up" of emotions can lead to unstable or depressed moods (Byrne, 2000). Therefore, the use of an expressive writing task can help an individual finally address some of the anxiety-inducing events that have plagued his or her subconscious, and while it may be emotionally difficult and result in immediate increases in anxiety, the overall catharsis of dealing with these events should regain some locus of control, decreasing the anxiety levels both in the short and long term.

\section{Present Study}

This study was part of a broader study that was examining the efficacy of Psychological First Aid (PFA) compared to Expressive Writing. The purpose of this study was to examine how the use of self-esteem words through an Expressive Writing task buffers anxiety levels across time compared to a control condition.

\section{Methodology}

\section{Participants}

The subjects $(N=31)$ who volunteered to participate in this study were Andrews University students who were 18 years of age or older and were from the Behavioral Science Research Subjects Pool. Subjects were compensated \$15 for their participation and also received research participation credit.

The study consisted of $19.4 \%$ male subjects and $80.6 \%$ females subjects. In the control "Superficial/Daily Writing" condition, $7.7 \%$ of the subjects were male, while a drastic 92.3\% of the subjects were female. In the "Expressive Writing" condition, 27.8\% of subjects were male, while $72.2 \%$ of subjects were female. In terms of class standing, $51.6 \%$ of the subjects were freshmen, $27.0 \%$ of the subjects were sophomores, $12.1 \%$ of the subjects were juniors, and $9.3 \%$ of subjects were seniors. The majority of subjects were single $(74.2 \%)$, followed by dating 
$(22.6 \%)$, and a minority of divorced (3.2\%). In terms of ethnicity, the three highest frequencies were Asian (22.6\%), White (22.6\%), and Multi-ethnic (22.6\%), followed by Latino (16.1\%), African American (3.2\%), and West Indian (3.2\%). Religious affiliation was largely Seventh-day Adventist (90.8\%), followed by none (6.5\%), and other $(2.7 \%)$.

\section{Measures}

The subjects were first given the demographic questionnaire to obtain information on age, gender, ethnicity, religious affiliation, and how often they engage in particular religious practices and behaviors.

State-Trait Anxiety Inventory, S-Anxiety Scale (STAI-S). The STAI-S (Spielberger et al., 1983) is a 20-item self-report questionnaire that assesses one's state anxiety, which is not linked to a person's natural inclination to worry or stress, but rather, what kind of stressors are currently affecting the subject at this moment. This survey was designed as a 4-point Likert scale, ranging from " $1=$ Almost Never" to " $4=$ Almost Always". For example, subjects must respond to how they relate to the statements: "I am presently worrying over possible misfortunes" or "I wish I could be as happy as others seem to be". The reliability coefficient of the STAI-S is rather low, around $r=.33$. The reason for this is that the questions should tease apart how the individuals are feeling at that moment, and not that they always react the same way. The scale was found to have concurrent validity, meaning it can predict outcomes, convergent validity, or that the multiple variables are related, divergent validity, or that it can show when two variables are not related, and construct validity, or the degree to which it measures what it says it is measuring (Spielberger et al., 1983).

Linguistic Inquiry and Word Count (LIWC). The LIWC program (Pennebaker, Boyd, Jordan, \& Blackburn, 2015) was used to analyze self-esteem from the raw text that subjects 
produced in regards to a stressful experience in their life through the Expressive Writing exercise. This program analyzes each word in a text document, compares it with the meaning of the sentence, then the paragraph, and creates multiple regressions to determine the most probable meaning of the word, and tallies the totals of each word into the summation of variables divided by the total number of words (Pennebaker et al., 2015). For this study, self-esteem was measured through LIWC. However, because there is no explicit "Self-Esteem" variable in LIWC, a strong literature review and understanding of the coefficients led to the use of "Clout" as an indicator for Self-Esteem. Clout itself is a measure of the writer's confidence, leadership status, or social status that is expressed through their writing. Most of the literature reviews do not give insight into how or why they chose certain coefficients, but what can be known from the literature as well as great deliberation, clout was used as a proxy for self-esteem. The reliability was good ( $r$ $=.58$ ) and that, based on personal correlational studies, LIWC was found to measure positive and negative emotions, cognitive strategies, themes and motifs, and language composition elements (Pennebaker et al., 2015).

\section{Group Conditions}

Subjects were randomly assigned into one of the two following conditions in the study: Expressive Writing (experimental) and Superficial Writing (control).

Expressive Writing Condition $(\mathbf{E W})$ : The EW condition is characterized by the prompt that was given to the subjects in this condition. After completing the demographic and baseline surveys, subjects were asked to think about a traumatic experience that they had gone through, and they were to write about it without stopping for 10 minutes. During this time, the instructor leaves the room, and begins a timer as soon as the subject begins to type. The researcher read the prompt out-loud to the subjects as they read it themselves, and it is as follows: 
For the next 10 minutes, I would like you to write your very deepest thoughts and feelings about a stressful life experience that has affected you and your life. In your writing, I'd like you to really let go and explore your deepest emotions and thoughts. You might tie your topic to your relationships with others, including parents, lovers, friends or relatives; to your past, your present or your future; or to who you have been, who you would like to be or who you are now. All of your writing will be completely confidential. Don't worry about spelling, grammar or sentence structure. The only rule is that once you begin writing, you continue until the time is up.

Superficial Writing Condition (SW): While similar to the expressive writing condition in most ways, the major difference, again, is the prompt given to the SW subjects. While subjects in the EW group were explicitly told to acknowledge and address their feelings about a stressful experience, the SW subjects were given a far less emotionally charged prompt that reads as follows:

For the next 10 minutes, I would like for you to write about how you have used your time over the past two weeks. In your writing, please go into as much detail as possible in how you have spent your days and managed your time. In your account of your activities, please be as objective as possible. You should describe your activities in detail without discussing any of your own thoughts or feelings related to the topic. All of your writing will be completely confidential. Don’t worry about spelling, grammar or sentence structure. The only rule is that once you begin writing, you continue until the time is up. The main purpose of utilizing prompts was to ensure that strong yet consistent emotional priming was given to the subjects of each condition. The EW prompt was read emphatically, with stronger swells and was said with a more reflective tone. The SW prompt was read just as 
emphatically, but was spoken with a rather calm inflection to ensure the SW subjects knew not to use any emotion within their writing.

\section{Procedure}

The parent study conducted a power analysis was conducted to determine the sample size necessary for this study. With an effect size of Cohen's $f=0.25$ and a power of .99 , a sample of 100 subjects would be necessary to study a moderate efficacy occurrence. To observe a moderate effect size, at least 50 subjects would be necessary for my study. Subjects were randomly assigned to either the Expressive Writing or to the Superficial Writing condition.

The study was made available online for subjects in the Behavioral Sciences Research Subjects pool. Subjects selected the study and then filled out the demographic questionnaire and read the consent form ahead of time. The participants specified a time that they were available for approximately 1 hour. During the scheduled time, subjects came in to the Behavioral Sciences Research Center Cog Lab 3, where they were greeted and situated. They then had the procedure explained to them, and informed consent was recorded. Each subject was then given a unique, randomly generated hash string so that no personal identifiers can be connected with their survey or written data.

Subjects then completed the STAI-S and all other measures associated with the study (see Appendix B) to establish baseline data. The subjects were then randomly assigned to one of the group conditions. After completing the condition, subjects completed all measures once again immediately afterwards, 30 minutes later, and 15 days later. After this session, the researcher thanked each participant, distributed and recorded that $\$ 15$ were paid, and gave two research credits to each subject for their completed participation. 
To analyze the data with the LIWC, the raw text would be cleaned up using Microsoft Word's auto-correct feature, as well as heuristic corrections, to minimize researcher engagement with the sensitive material, and also to ensure that appropriate coefficients could be determined. In addition, the data from the STAI-S would be calculated with simple means, and these mean scores would have their variance compared within subjects' multiple sessions and between the two groups, also known as a repeated measures ANOVA. Finally, a moderation analysis would be run on the anxiety scores, if a significant interaction between anxiety levels and self-esteem coefficients were found, to see how, if at all, the self-esteem of the individual affected the changes in anxiety.

\section{Results}

A repeated measures ANOVA was run both between and within subjects to determine if anxiety levels changed in subjects across time. A moderation analysis was to be used to determine if levels of self-esteem taken from LIWC had any moderating effects on the changing anxiety levels. This was then compared between the two groups to look for differences in anxiety level changes in the expressive writing group compared to the superficial writing group.

The within-group ANOVA showed a statistically significant correlation between time and anxiety $F(3,147)=8.92, p<.001$, (see Appendix D) for both the Expressive Writing and Superficial Writing conditions. In other words, all participants, regardless of group, showed decreases in anxiety levels across time. However, there was no significant interaction between the scores and which group the subjects were in $F(3,147)=1.07, p>.05$. Again, this means that, because both groups showed decreases in anxiety levels, it is not possible to say whether this decrease was due to being in either the EW or SW groups. Lastly, the between subjects ANOVA, which would look at the effect the treatment had on changes in anxiety levels, was not 
statistically significant $F(3,49)=1.87, p>.05$. Therefore, when comparing both groups, one group is not decreasing anxiety faster than the other. Because of this data, there is no reason to run a moderation analysis on self-esteem using the clout variable, as there is no difference in anxiety levels across time that would be moderated by self-esteem. In other words, self-esteem is not what is driving the changes in anxiety levels as the hypothesis had suggested.

To analyze the effects of self-esteem, a moderation analysis could not be run because of the lack of statistical significance. Therefore, a repeated measures ANOVA had to be run on data again. In order to analyze self-esteem, the subjects for each group (EW or SW) were organized by their clout coefficients from LIWC, which would be used to estimate their self-esteem levels. Once both the EW and SW groups were organized by clout coefficients, they groups were each cut in half to look for a difference between "high self-esteem" and "low self-esteem" groups both between and within subjects (see Appendix $E_{1}$ and $E_{2}$ ).

When separated, the group condition was still not significant $F(3,60)=3.37, p>.05$. Therefore, self-esteem did not contribute to the fact that the treatment did not affect anxiety levels. However, the relationship between anxiety and clout were significant $F(3,60)=5.962, p$ $<.001$, which means that subjects with higher levels of self-esteem showed lower levels of anxiety across time. However, clout levels were not significantly different across subjects $F$ (1, $20)=1.69, p>.05$. Because of this, while clout may affect changes in anxiety levels, levels of clout were not significantly different between subjects.

\section{Discussion}

The current study sought to determine if anxiety levels over time decreased when presented with an expressive writing condition, and if this change in anxiety levels was moderated by one's level of self-esteem. After conducting the mixed measures between-subjects 
and within-subjects ANOVAs, there was a significant decrease in all participant's anxiety levels across time, however, the expressive writing condition and the self-esteem levels were not the ones driving that change. The null hypothesis was that the changes in anxiety levels would not be affected by the expressive writing condition, and that those who were in the expressive writing condition would show the same effects as those in the superficial writing condition. In addition, the null hypothesis stated that self-esteem levels would have no effect on the changes to anxiety levels across time. On the other hand, the alternative hypothesis was that anxiety levels would remain relatively stable for the daily writing group, and that the anxiety levels of the expressive writing group would show increases in anxiety levels between the first and second sessions, but would lead to decreasing levels of anxiety, with those with higher self-esteem would show longer, steeper decreases in anxiety. In addition, the alternative hypothesis also stated that high self-esteem levels would show a moderating effect by decreasing anxiety levels overall, as compared to low self-esteem participants. Therefore, the current study failed to reject the null hypothesis.

While the alternative hypothesis was not supported, there was the interesting and significant pattern that all subjects showed decreases in their anxiety levels, both in the short term and the long term (see Appendix C for graph). One possible explanation for this is that, due to the length of the 15-day posttest, many participants performed their first three sessions before Spring Break, when there are typically many tests and assignments due before the Break, and when the 15-day posttest came around after Spring Break, the participants had not been stressed because they were enjoying their break instead of worrying about academics. While this hypothesis may explain why the final session would show lower levels of anxiety, it would not explain why there tends to be a decrease in anxiety levels from session one to session two and 
three. Therefore, it is possible to infer that perhaps not all of the variance is accounted for within the measures of this study, which is possible, as the STAI-S can only give quantitative, selfreported information about the subject's anxiety levels.

Finally, in terms of self-esteem, it is likely that self-esteem does have an effect on changes in anxiety levels, the level of self-esteem necessary for each person is different meaning that measuring how many self-esteem related words a subject uses is not sufficient for understanding how self-esteem affects each subject.

\section{Limitations}

This study does contain quite a few limitations that likely could have decreased the power and the validity of the study. First and foremost, the study was underpowered, meaning that it was not able to reach $N=50$, as this would be the minimum participant count required to even consider a moderate effect size between the two groups. In addition, the moderation analysis that was planned for the data could not be run, due to the lack of statistical significance in the time * group interaction (see Appendix D for the data). In addition, while self-esteem could have led to steeper decreases in anxiety, it is also possible that self-esteem serves to lower the baseline anxiety that an individual experiences, rather than how quickly or persistently their anxiety levels decrease.

In addition, it is important to note that the study's methodology was fixed to the specifications required by the IRB, as the study itself was ethically reviewed as a portion of a larger, ongoing study on mood and resilience in college students, and how Psychological First Aid can help. Therefore, no changes could be made at this time, to preserve the full requirements of both this study and the parent study. 
To make matters even murkier, Pennebaker does not share how the variables in LIWC are encoded, as it is a paid software, and any explanation beyond the fact that it has strong validity could lead to the undoing of his work and profit, which proves problematic. Finally, there is always the variance associated with human beings, as this study was designed around human subjects who are to go about their day and live different experiences, and then to come and self-report their current state anxiety correctly, as well as adequately express and establish themselves in a short writing exercise. A strong suggestion would be to redo the entire study based off of more research on expressive writing, and to continue the study to obtain enough subjects to prove that the lack of results in not due to chance. The variable of clout, in addition, would also be far more stable if given several raw texts to be compared between. That would allow internal validity to be established to make sure that a subject would have used a large amount of clout words due solely to chance.

A final note is that the EW participants were asked to recall a stressful event, which is extremely different from having physically, mentally, and psychologically having just gone through a traumatic experience. Therefore, changes in anxiety levels likely vary greatly from other PFA or even Pennebaker's studies, due to the nature of the populations being sampled. In addition, while the SW were asked to perform what is believed to be an emotional-neutral task, the recounting of how one has spent his or her time can also be stressful, especially to college students who spend a great deal of their time either working on academic projects or attempting to not think about academic projects, which could skew their state anxiety scores.

\section{Implications}

Anxiety will always be a part of the human condition. It serves a fundamental, biological purpose in formulating goal-oriented answers to the problems in life. However, while anxiety 
can serve a purpose, it can often begin to run rampant in ways that can damage quality of life. Therefore, anxiety has to be controlled, not eradicated. While it is unclear whether creative writing has a profound effect on anxiety levels when presented as a single activity, perhaps there is much more merit to continual, effortful expressive writing. Perhaps there truly is an effect that will be teased apart through more accurate means. While this study has failed to support the alternative hypothesis, there is always the requirement for strong, methodical replication studies with statistically powerful $N$ values. Perhaps future research will continue to qualify and establish how to detect and decrease anxiety. 


\section{References}

American Psychiatric Association. Committee on Civil Defense. (1954). Psychological first aid in community disasters. Washington, DC.

Bonanno, G. A. (2008). Loss, trauma, and human resilience: Have we underestimated the human capacity to thrive after extremely aversive events? Psychological Trauma: Theory, Research, Practice, and Policy, 1, 101-113.

Blackburn, K., Boyd, R. L., Jordan, K., \& Pennebaker, J. W. (2015). The development and psychometric properties of LIWC2015. Austin, TX: University of Texas at Austin.

Brymer, M., Jacobs, A., Layne, C., Pynoos, R., Ruzek, J., Steinberg, A., Vernberg, E., \& Watson, P. (2006). Psychological first aid: Field operations guide (2nd ed.) National Child Traumatic Stress Network and National Center for PTSD.

Burling, J., Greenberg, J., Lyon, D., Pinel, E., Pyszczynski, T., Rosenblatt, A., Simon, L., \& Solomon, S. (1992). Why do people need self-esteem? Converging evidence that self-esteem serves an anxiety-buffering function. Journal of Personality and Social Psychology, 63, 913-922. doi:10.1037/0022-3514.63.6.913

Byrne, B. (2000). Relationship between anxiety, fear, self-esteem, and coping strategies in adolescence. Adolescence, 35, 201-215.

David, D. (2011). Resilience as a protective factor against compassion fatigue in trauma therapists. Unpublished doctoral dissertation. Walden University: Minneapolis, MN.

De Jong, P. J. (2001). Implicit self-esteem and social anxiety: Differential self-favouring effects in high and low anxious individuals. Behavior and Research Therapy, 40, 501-508.

Everly, G.S., Welzant, V., \& Jacobson, J. M. (2008). Resistance and resilience: The final frontier 
in traumatic stress management. International Journal of Emergency Mental Health, $10(4), 261-270$.

Koivula, N., Hassmen, P., \& Fallby, J. (2000). Self-esteem and perfectionism in elite athletes: Effects on competitive anxiety and self-confidence. Personality and Individual Differences, 32, 865-875.

Pennebaker, J. W. (1990). Opening up: The healing power of expressing emotions. New York: The Guilford Press.

Robinson, R. L., Navea, R., \& Ickes, W. (2013). Predicting final course performance from Students' written self-introductions: A LIWC analysis. Journal of Language and Social Psychology, 32, 469-479. doi: 10.1177/0261927X13476869

Rutter, M. E. (1987). Psychosocial resilience and protective mechanisms. American Journal of Orthopsychiatry, 57, 316-331.

Stewart, A. J. \& McDermott, C. (2004). Gender in psychology. Annual Review of Psychology, $55,519-544$.

Tausczik, Y.R., \& Pennebaker, J.W. (2010). The psychological meaning of words: LIWC and computerized text analysis methods. Journal of Language and Social Psychology, 29, 24-54.

Taylor, D. N. (1995). Effects of a behavioral stress-management program on anxiety, mood, self-esteem, and T-cell count in HIV-positive men. Psychological Reports, 76, 451-457. 
Appendix A

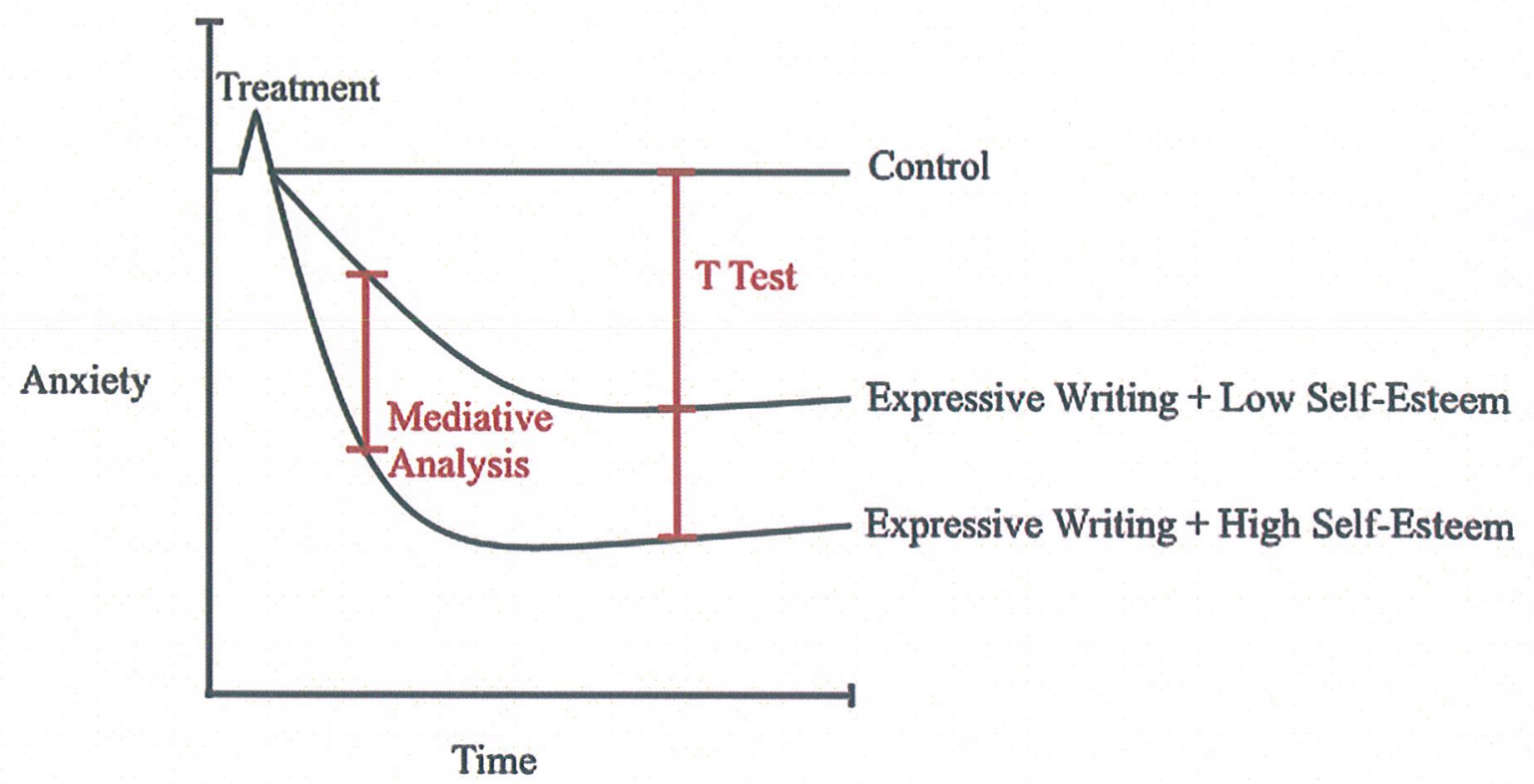

Figure 1. Visual representation of the hypothesis of stress levels. Due to a typo, the analysis says "Mediative Analysis". In truth, it was a moderation analysis that would be run to identify a difference in anxiety levels due to self-esteem. 
Appendix B

\begin{tabular}{|c|l|l|l|l|l|}
\hline Group & Baseline & Condition & After Exposure & Post 30-minutes & Post 15-days \\
\hline 1 & $A^{*}, \mathrm{~B}, \mathrm{C}, \mathrm{D}$ & Expressive Writing & A*, B, C, D & A*, B, C, D & A*, B, C, D \\
\hline 2 & A*, B, C, D & Superficial Writing (control) & A*, B, C, D & A*, B, C, D & A*, B, C, D \\
\hline
\end{tabular}

$\mathrm{A}^{*}=$ STAI-S, B = POMS, C = RSES, D = GSR

Table 1. Road map of the surveys that the subjects completed. Only the STAI-S value would be analyzed in this study. They were compared to the clout coefficients, which were obtained during the condition. 
Appendix C

TIME * GROUP

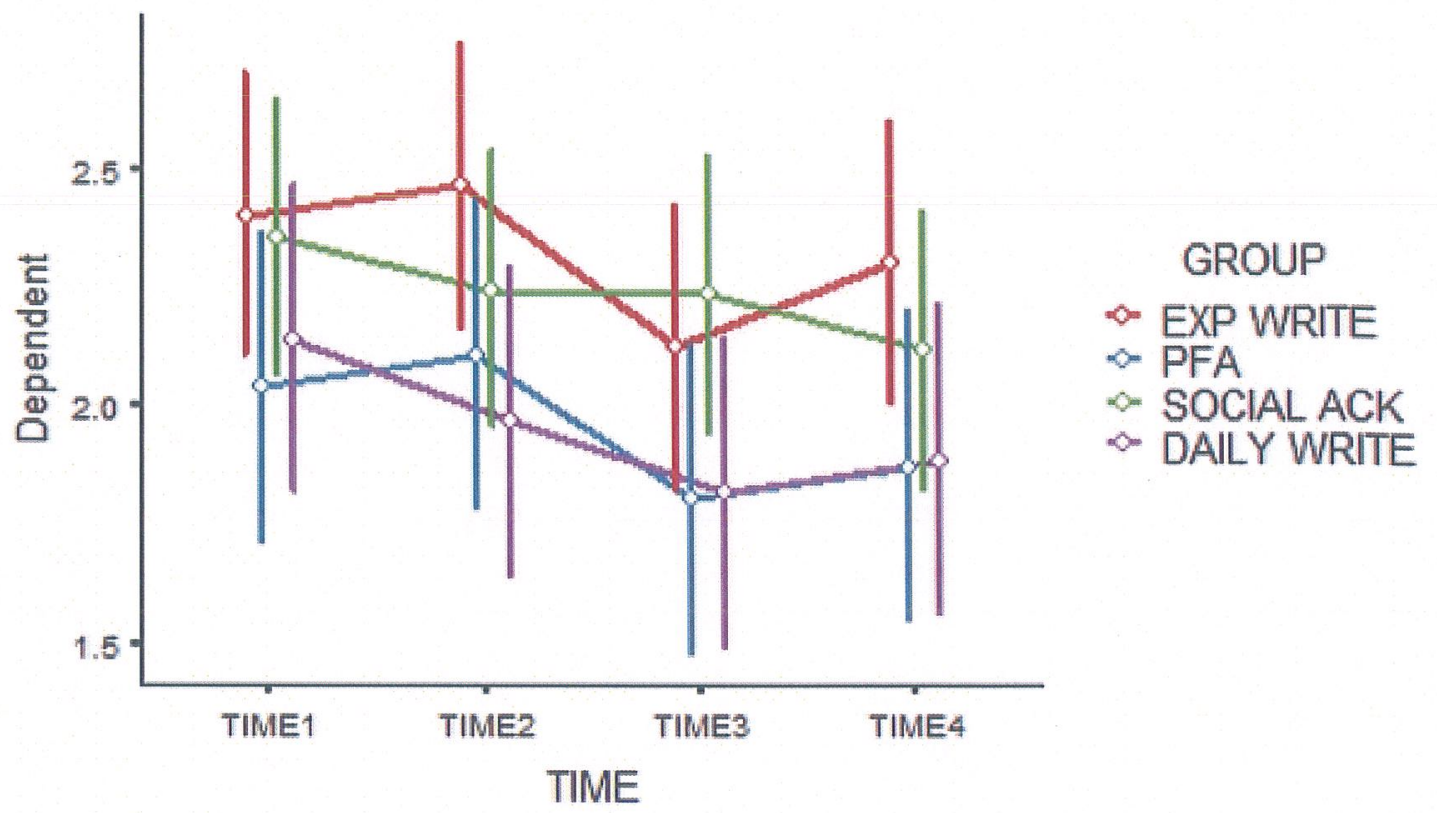

Figure 2. A graph of the levels of anxiety (dependent) against time. This study only dealt with the red and purple lines, the other two groups are part of the broader parent study. 
Appendix $\mathrm{D}_{1}$

\section{Repeated Measures ANOVA}

\begin{tabular}{lrrrrr}
\hline \multicolumn{1}{l}{ Within Subjects Effects } \\
\hline & Sum of Squares & df & Mean Square & \multicolumn{1}{c}{ F } & P \\
\hline TIME & 2.068 & 3 & 0.6892 & 8.92 & $<.001$ \\
TIME *: GROUP & 0.741 & 9 & 0.0824 & 1.07 & 0.391 \\
Residual & 11.354 & 147 & 0.0772 & & \\
\hline
\end{tabular}

Note. Type 3 Sums of Squares

Table 2. Within Subjects ANOVA data. The first line shows the changes in anxiety levels across time for all of the subjects. The variance between time and anxiety was significant $F(3,147)=$ $8.92, p<.001$. The significance means that anxiety levels in all subjects decreased over time. The second line shows the changes in anxiety levels across time within each group. The variance is not significant $F(3,147)=1.07, p>.05$; therefore, the subjects' changes in anxiety were not affected by which group they were in (EW or SW). 


\section{Appendix $\mathrm{D}_{2}$}

\begin{tabular}{lrrrrr}
\multicolumn{6}{l}{ Between Subjects Effects } \\
\hline & Sum of Squares & df & Mean Square & $F$ & $\mathrm{p}$ \\
\hline GROUP & 5.66 & 3 & 1.89 & 1.87 & 0.146 \\
Residual & 49.36 & 49 & 1.01 & & \\
\hline
\end{tabular}

Note: Type 3 sums of Squares

Table 3. Between Subjects ANOVA data. The first line is looking to see if anxiety levels were being driven by which group the participants were in, EW or SW. No significance was obtained $F(3,49)=1.87, p>.05$; therefore, anxiety levels were roughly the same between both groups, meaning that the treatment did not decrease anxiety levels beyond the norm. 
Appendix $E_{1}$

\begin{tabular}{|c|c|c|c|c|c|}
\hline & Sum of Squares & $d f$ & Mean Square & $\mathrm{F}$ & $\mathrm{P}$ \\
\hline Anxiety & 2.230 & 3 & 0.743 & 3.371 & 0.024 \\
\hline Anxiety s: CONDITION & 0.625 & 3 & 0,208 & 0.945 & 0.425 \\
\hline Anxiety : CLOUT & 3.944 & 3 & 1.315 & 5.962 & 0.001 \\
\hline Residual & 13.230 & 60 & 0.221 & & \\
\hline
\end{tabular}

Note. Type 3 Sums of Squares

Table 4. Within Subjects ANOVA data when accounting for self-esteem. The first line looks at if anxiety levels are consistent throughout all scores. No significance was obtained $F(3,60)=$ $3.37, p>.05$; therefore, anxiety levels were not staying the same - they were decreasing across time. The second line looks to see if changes in anxiety levels were being driven by which condition (EW or SW) the subject was in. No significance was obtained $F(3,60)=0.945, p>$ .05 ; therefore, the treatment still did not account for the changes in anxiety levels. The third line asks if changes in anxiety levels are related to self-esteem levels, as accounted for as clout. The variance between changes in anxiety levels and self-esteem were significant $F(3,60)=5.962, p$ $<.001$; therefore, regardless of the group, individuals with higher levels of clout showed lower levels of anxiety. 
Appendix $\mathrm{E}_{2}$

\begin{tabular}{lccccc}
\hline \multicolumn{1}{l}{ Between Subjects Effects } \\
\hline & Sum of Squares & df & Mean Square & F & p \\
\hline CONDITION & 3.15 & 1 & 3.15 & 2.47 & 0.132 \\
CLOUT & 2.15 & 1 & 2.15 & 1.69 & 0.209 \\
Residual & 25.52 & 20 & 1.28 & & \\
\hline
\end{tabular}

Note. Type 3 Sums of Squares

Table 5. Between Subjects ANOVA data when accounting for self-esteem. The first line asks if there was a difference in anxiety levels between the EW and SW groups. No significance was obtained $F(1,20)=2.47, p>.05$; therefore, the treatment still did not drive the changes in anxiety levels. The second line looks at if subjects showed significantly different levels of clout. No significance was obtained $F(1,20)=1.69, p>.05$; therefore, between subjects, levels of clout were relatively similar. 